\title{
Interlayer Structure of Bioactive Molecule, 2-Aminoethanesulfonate, Intercalated into Calcium-Containing Layered Double Hydroxides
}

\author{
Tae-Hyun Kim, Hyoung Jun Kim, and Jae-Min Oh \\ Department of Chemistry \& Medical Chemistry, College of Science \& Technology, Yonsei University, Wonju, \\ Gangwon-do 220-710, Republic of Korea \\ Correspondence should be addressed to Jae-Min Oh, jaemin.oh@yonsei.ac.kr
}

Received 14 February 2012; Accepted 5 April 2012

Academic Editor: Krasimir Vasilev

Copyright () 2012 Tae-Hyun Kim et al. This is an open access article distributed under the Creative Commons Attribution License, which permits unrestricted use, distribution, and reproduction in any medium, provided the original work is properly cited.

We have successfully intercalated 2-aminoethanesulfonate, a well-known biomolecule taurine, into calcium-containing layered double hydroxides via optimized solid phase intercalation. According to X-ray diffraction patterns and infrared spectroscopy, it was revealed that the intercalated taurine molecules were each directly coordinated to other calcium cation and arranged in a zig-zag pattern. Scanning electron microscopy showed that the particle size and morphology of the LDHs were not affected by the solid phase intercalation, and the surface of intercalates was covered by organic moieties. From ninhydrin amine detection tests, we confirmed that most of the taurine molecules were well stabilized between the calcium-containing LDH layers.

\section{Introduction}

Drug delivery systems are now a major concern in nanoscience and nanotechnology [1-5], and the two-dimensional nanomaterial layered double hydroxide (LDH) is attracting increasing interest in the field. LDHs have a general chemical formula of $\left[\mathrm{M}(\mathrm{II})_{1-x} \mathrm{M}(\mathrm{III})_{x}(\mathrm{OH})_{2}\right]^{x+}\left(\mathrm{A}^{n-}\right)_{x / n} \cdot \mathrm{mH}_{2} \mathrm{O}$ (M(II), M(III): metal ions, $\mathrm{A}^{n-}$ anionic species). They are composed of positively charged layers and exchangeable interlayer anions along water molecules [6]. Their crystal structure evolves from electrically neutral brucite$\left(\mathrm{Mg}(\mathrm{OH})_{2-}\right)$ like layers in which $\mathrm{M}(\mathrm{II})(\mathrm{OH})_{6}$ octahedrons are connected in the $x y$-plane direction by sharing their edges. The isomorphic substitution of $\mathrm{M}$ (II) with $\mathrm{M}$ (III) causes a positive charge on the layers, and the anionic species between the layers compensate and produce charge neutrality. The interlayer anions, which can be a number of diverse things from small molecules to drugs or DNA strands, can acquire stabilization energy through electrostatic interaction with the layers [7-9].

For decades, LDH nanomaterials, have been widely studied as antacids, catalytic supports, polymer stabilizers, adsorbents and others [6, 10-12]. The last ten years, however, have been more devoted to studying LDHs for biomedical applications due to their tailored properties as drug delivery nanocarriers, ability to stabilize interlayer anions from external harsh conditions $[7,13]$, high rate of cellular uptake via clathrin-mediated endocytosis $[14,15]$, low toxicity due to dissolution physiological $\mathrm{pH}[16,17]$, and easily modifiable surface for additional functionality [18].

The intercalation of biologically active substances such as anticancer or anti-inflammatory drugs and therapeutic genes into LDHs has been reported to dramatically enhance the curative efficacy as well as to release the drug molecules in sustained manner $[9,19]$.

Among the various types of LDHs with different metal compositions, LDHs containing calcium are unique in their structure and expected to be highly biocompatible. As reported, biological systems, especially human bodies, have abundant calcium moieties for the formation of skeletal structure and for the cell signaling [20], so it follows that LDHs with $\mathrm{Ca} / \mathrm{Al}$ or $\mathrm{Ca} / \mathrm{Fe}$ compositions should be highly biocompatible as well. The structure of calcium-containing LDHs (abbreviated as CaM-LDHs) is different from others 


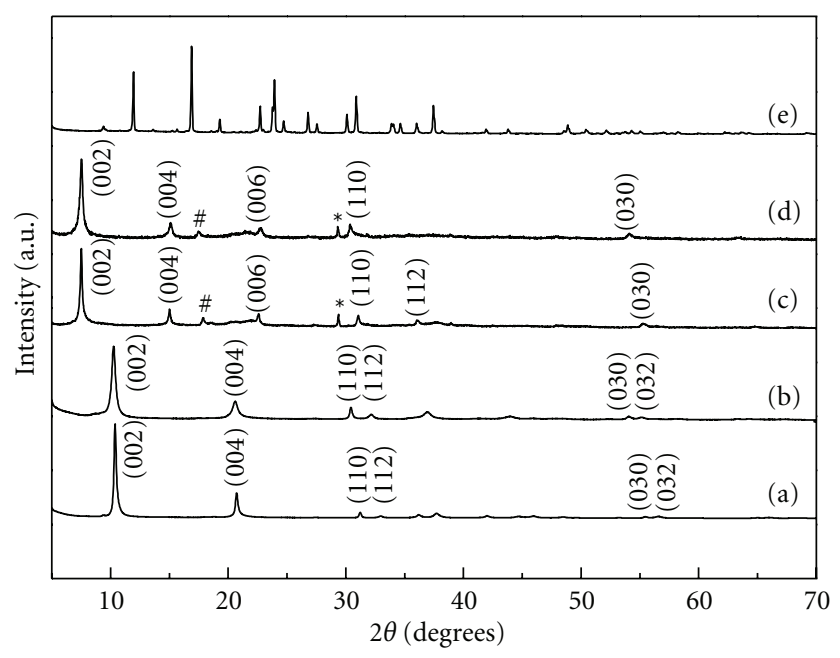

Figure 1: X-ray diffraction patterns for (a) CaAl-LDH, (b) CaFe$\mathrm{LDH}$, (c) CaAl-Tau-LDH, (d) CaFe-Tau-LDH, and (e) $\mathrm{Na}^{+}$-taurine salt. ( ${ }^{*}: \mathrm{NaNO}_{3},{ }^{*}$ : undetermined impurity).

due to the large size of the $\mathrm{Ca}^{2+}$ ions. Heptacoordinated calcium hydroxide decahedrons and hexacoordinated trivalent metal hydroxide octahedrons are arranged in a 2dimensional lattice by sharing their edges, however, the seventh coordination on calcium is usually occupied by interlayer anions or water molecules [21].

Despite the expected advantages in drug delivery applications, there have only been a few reports on the intercalation of biologically active molecules into CaM-LDHs. The possible bond between metal cations and anions during the synthesis may prevent the formation of anion-intercalated $\mathrm{LDH}$ in coprecipitation, which is the most well-known synthesis method for LDH. In ion exchange reactions, the dissolution of layers rather than intercalation may occur due to the dissolution properties of CaM-LDHs in neutral $\mathrm{pH}$.

In this study, we successfully intercalated the bioactive organic acid taurine (2-aminoethanesulfonate) into CaAl- or CaFe-LDHs via solid phase reaction. Taurine (Tau) is a wellknown bile product that acts in antioxidation and osmoregulation [22] and recently has been attracting gaining interest for drug delivery systems [23]. We demonstrate systematic approaches for solid phase intercalation of taurine molecules into CaM-LDHs as an alternative for coprecipitation and ion exchange routes. The structural analyses of taurineintercalated CaM-LDHs are described in terms of interlayer structure and bonding nature.

\section{Experimental and Procedures}

2.1. Materials. $\mathrm{Ca}\left(\mathrm{NO}_{3}\right)_{2} \cdot 4 \mathrm{H}_{2} \mathrm{O}$ was purchased from Junsei Chemical Co., Ltd., (Tokyo, Japan); $\mathrm{Al}\left(\mathrm{NO}_{3}\right)_{3} \cdot 9 \mathrm{H}_{2} \mathrm{O}$, $\mathrm{Fe}\left(\mathrm{NO}_{3}\right)_{3} \cdot 9 \mathrm{H}_{2} \mathrm{O}$, and $2 \%$ ninhydrin solution were purchased from Sigma-Aldrich Co., Ltd. (USA); $\mathrm{NaOH}$ pellets were obtained from Daejung Chemicals \& Metals Co., Ltd., (Gyonggido, Korea) and used without further purification. Taurine (2-aminoethanesulfonate) was purchased from Sigma-Aldrich Co., Ltd., (USA), and $\mathrm{Na}^{+}$-taurine salt was prepared by mixing taurine and $\mathrm{NaOH}$ solution with $1: 1$ molar ratio followed by drying with a rotary evaporator.

2.2. Synthesis of Pristine CaM-LDHs and Solid Phase Intercalation. For the preparation of CaAl- and CaFe-LDHs mixed metal nitrate solutions $\left(0.315 \mathrm{M}^{\text {of }} \mathrm{Ca}^{2+}\right.$ and $0.158 \mathrm{M}$ of $\mathrm{M}^{3+}\left(\mathrm{Al}^{3+}\right.$ or $\left.\mathrm{Fe}^{3+}\right)$ ) were prepared and titrated with $\mathrm{NaOH}$ solution until the $\mathrm{pH}$ reached $\sim 11.5$ and $\sim 13.0$, respectively. The precipitates were aged for 24 hours under $\mathrm{N}_{2}$ atmosphere with vigorous stirring. The products were filtered and washed with decarbonated water and dried in vacuum at $40^{\circ} \mathrm{C}$. Pristine LDHs $(0.2 \mathrm{~g})$ were mixed with taurine salts $(0.0802 \mathrm{~g}$ for CaAl- and $0.0762 \mathrm{~g}$ for CaFe-LDH to achieve a $1: 1$ molar ratio between taurine and $\mathrm{M}^{3+}$ ) and ground in a mortar for $5 \mathrm{~min}$ with various amounts of water added $(0,5,10,20,30$, and $40 \mu \mathrm{L})$. After grinding, the products were dried for 12 hours in vacuum at $40^{\circ} \mathrm{C}$.

2.3. Characterization. Powder X-ray diffraction patterns were obtained with a Bruker AXS D2 Phaser with degree and time step increments of $0.02^{\circ}$ and $1 \mathrm{sec} / \mathrm{step}$, respectively. Fourier transform infrared (FT-IR; Perkin Elmer, spectrum one B.v5.0) spectroscopy was performed with conventional $\mathrm{KBr}$ methods. The particle size and morphology of the CaM-LDHs and corresponding intercalates were investigated with scanning electron microscopy (SEM) on a Quanta 250 FEG at Yonsei University in Wonju. The chemical compositions of both LDHs were evaluated with inductively coupled plasma-atomic emission spectroscopy (ICP-AES: Perkin Elmer Optima-4300 DV) and elemental analysis (EA: EA 1110).

2.4. Primary Amine Detection (Ninhydrin Test). For ninhydrin test, CaAl-, CaFe-LDHs, taurine salt, CaAl-Tau-, and CaFe-Tau-LDHs were dispersed in $2 \%$ ninhydrin solution and diluted with ethanol at four-times the volume. After vortexing for $1 \mathrm{~min}$, aliquot was gathered and redispersed in ethanol for UV-Vis spectroscopy (UV-1800; SHZMADZU) and photographs.

\section{Result and Discussion}

In order to intercalate taurine molecules into CaM-LDH, we first tried the most widely utilized intercalation routes, coprecipitation and ion exchange reactions. As indicated in the X-ray diffraction patterns (see Figure S1 in Supplementary material available online at doi: 10.1155/2012/987938), coprecipitation was determined not to be effective for the preparation of taurine intercalated CaM-LDHs (CaM-TauLDHs). In the coprecipitation reaction system, there were 7-times as much nitrate as taurine molecules; therefore, nitrate ion is favored for intercalation. The ion exchange was also proven not to be adaptable for the intercalation of taurine into CaM-LDHs, although there were 1.5-times more taurine molecules than nitrate (data not shown). Studies on intercalation utilizing CaM-LDHs through coprecipitation and ion exchange have been less reported compared with general LDHs, and different types of intercalation such as 

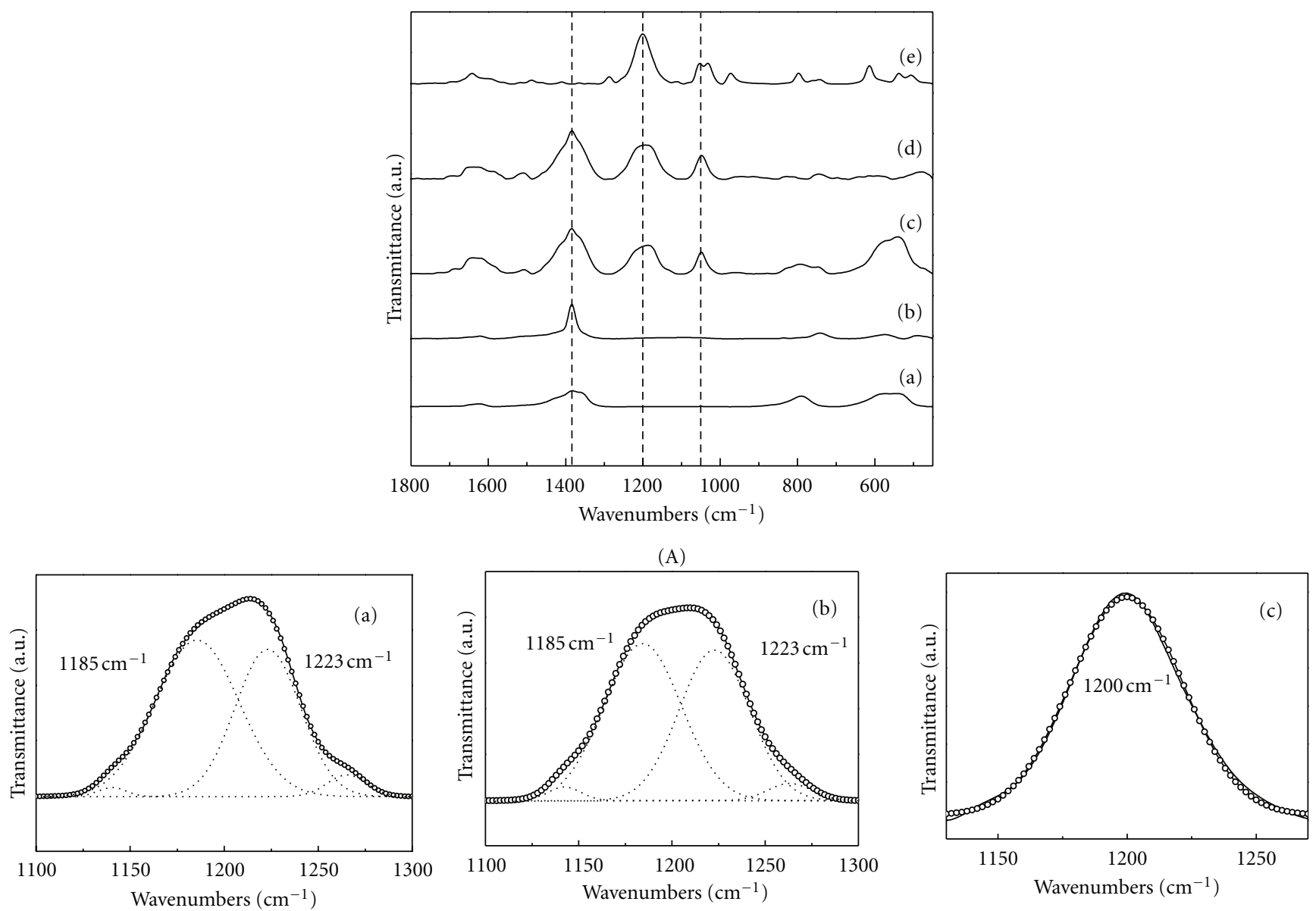

(B)

Figure 2: (A) Fourier transform infrared spectra for (a) CaAl-LDH, (b) CaFe-LDH, (c) CaAl-Tau-LDH, (d) CaFe-Tau-LDH, and (e) Na ${ }^{+}$taurine salt. (B) The magnified and multipeak fitted $\nu_{\text {asym }}\left(\mathrm{SO}_{3}{ }^{-}\right)$: (a) CaAl-Tau-LDH, (b) CaFe-Tau-LDH, and (c) Na ${ }^{+}$-taurine salt. (Solid line: observed peak, dotted line: fitted peaks, open circle: summation of fitted peaks).

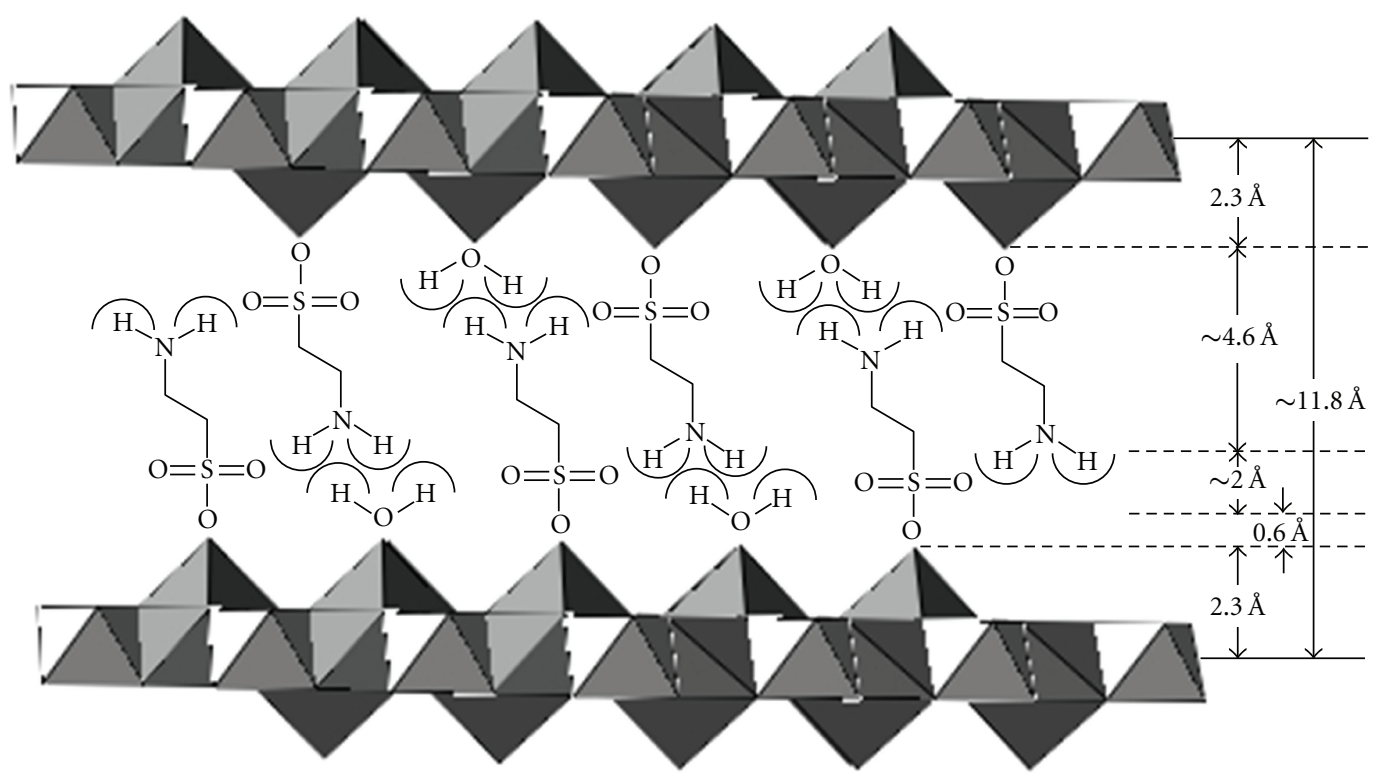

FIGURE 3: Schematic diagram for the possible orientation of interlayer taurin molecules in the CaM-Tau-LDH hybrid. 


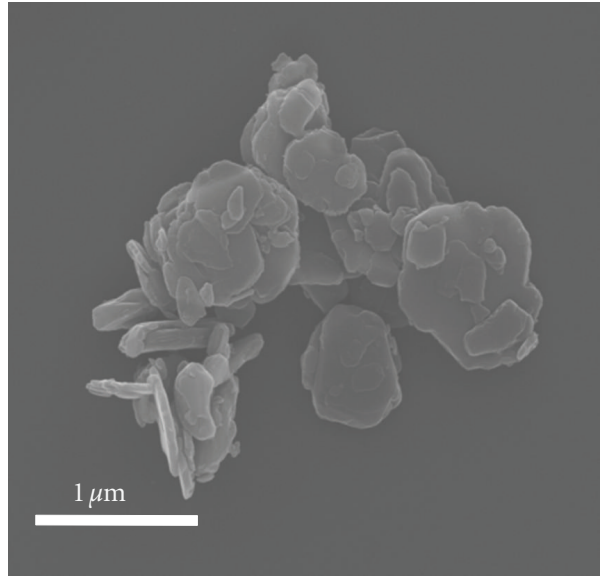

(a)

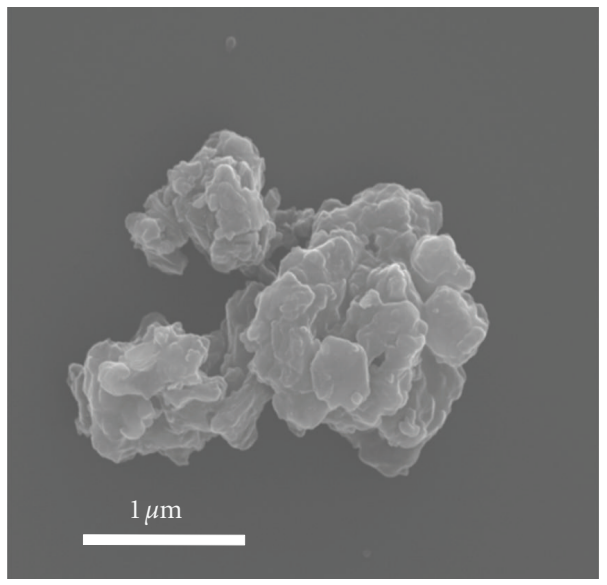

(c)

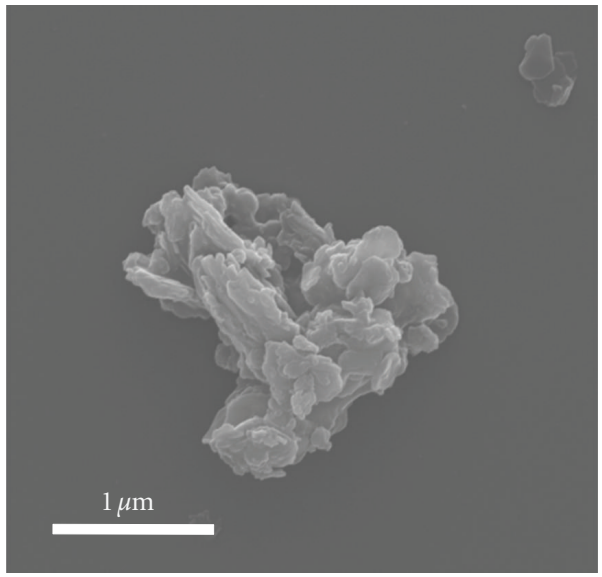

(b)

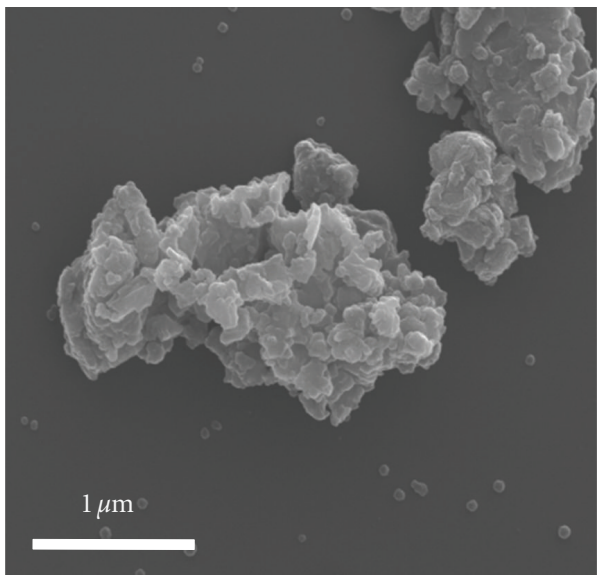

(d)

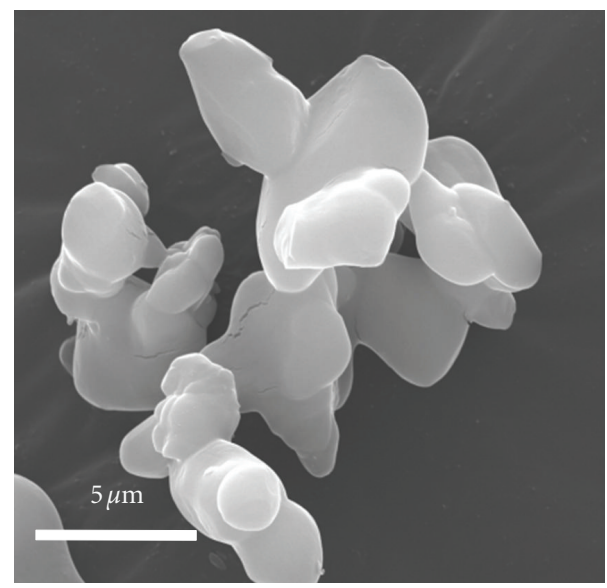

(e)

Figure 4: Scanning electron microscopic images for (a) CaAl-LDH, (b) CaFe-LDH, (c) CaAl-Tau-LDH, (d) CaFe-Tau-LDH, and (e) Na ${ }^{+}-$ taurine salt.

induced-hydrolysis-based reactions have been studied by Plank and von Hoessel [24].

The best synthetic strategy intercalation of taurine into CaM-LDHs was solid phase intercalation, in which both pristine LDHs and anion molecules are homogeneously ground in mortars with only a small amount of water. Since the amount of water is a key synthetic condition in this solid phase intercalation [25], various amounts of water were added to find an optimum condition (Figures S2 and S3). Although a phase transformation correlation with 

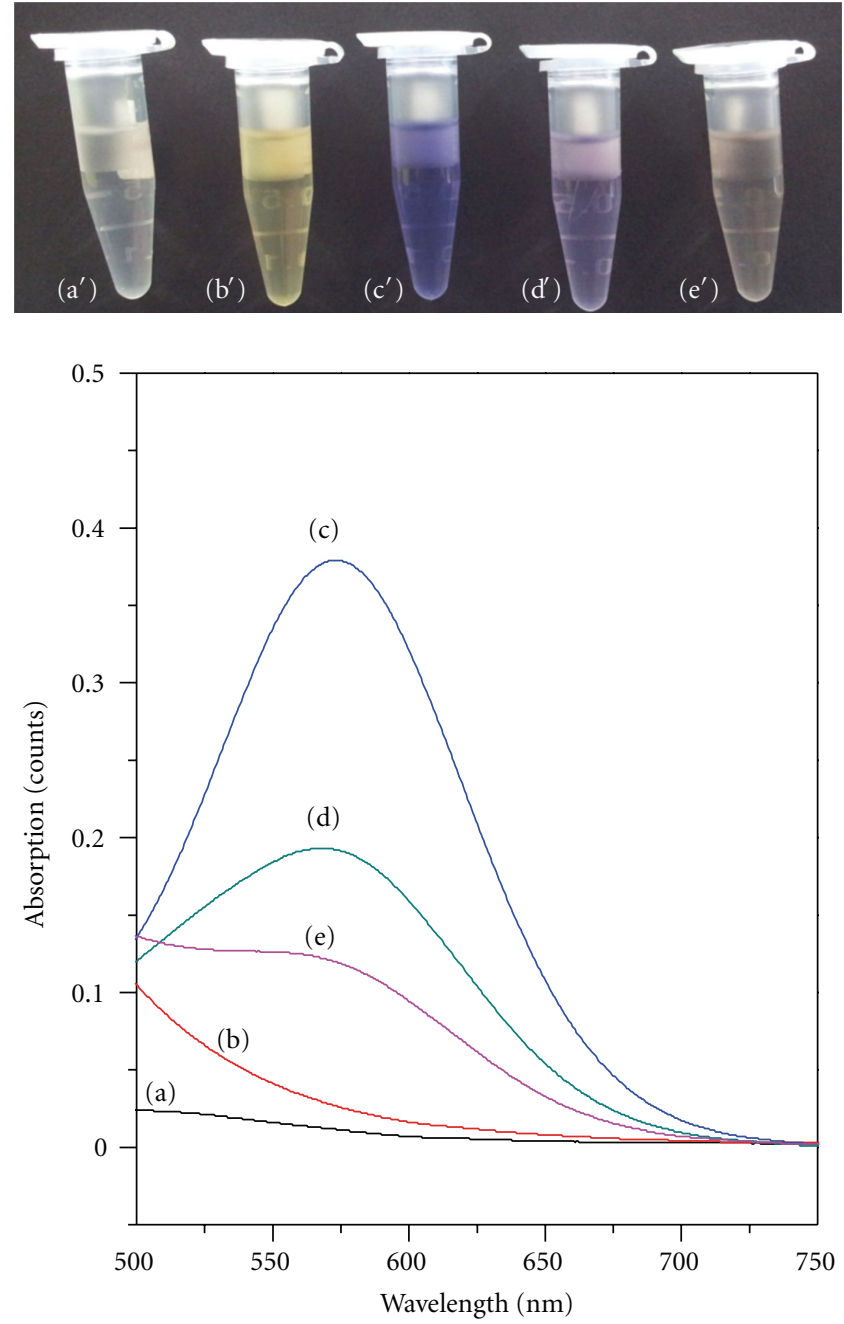

FIGURe 5: UV-Vis spectra for ninhydrin-treated (a) CaAl-LDH, (b) $\mathrm{CaFe}-\mathrm{LDH}$, (c) $\mathrm{Na}^{+}$-taurine salt, (d) CaAl-Tau-LDH, and (e) CaFeTau-LDHs. The inset shows photographs for ninhydrin-treated $\left(\mathrm{a}^{\prime}\right)$ CaAl-LDH, ( $\left.b^{\prime}\right) \mathrm{CaFe}-\mathrm{LDH},\left(\mathrm{c}^{\prime}\right) \mathrm{Na}^{+}$-taurine salt, (d') CaAl-Tau$\mathrm{LDH}$, and $\left(\mathrm{e}^{\prime}\right) \mathrm{CaFe}-\mathrm{Tau}-\mathrm{LDH}$.

increasing amounts of water added could not be found, it was determined that the addition of $10 \mu \mathrm{L}$ water to $0.2 \mathrm{~g}$ CaM$\mathrm{LDH}$ and $\sim 0.08 \mathrm{~g} \mathrm{Na}^{+}$-taurine salt is the optimum synthetic condition.

Figure 1 shows the X-ray diffraction patterns for the CaM-LDHs and their taurine intercalates. The diffraction patterns for the pristine LDHs corresponded well with previously reported results (Figure 1) [21,26]. After intercalation, the (001) peaks shift to the lower 2-theta region, revealing lattice expansion from $\sim 8.5$ to $\sim 11.8 \AA$ along the $z$-axis (layer stacking direction). The lattice parameters $a$ and $c$ in the hexagonal crystal system for pristine material and intercalates were calculated based on the $(h k l)$ indexing results. The $c$ values increase from 17.12 and 17.25 to 23.58 and 23.44 after intercalation for CaAl- and CaFe-LDHs, respectively, while the $a$ values remain almost constant at $\sim$ 5.74 and $\sim 5.87 \AA$. This result revealed that the 2-dimensional lattice structures of the LDHs were neither decomposed nor dissolved during the intercalation. The asterisks indicate the diffraction patterns for $\mathrm{NaNO}_{3}$ salt which may have resulted from the disintercalated nitrates.

In order to verify the preservation of functional groups in taurine as well as the bonding nature between taurine and the LDHs, Fourier transform infrared (FT-IR) spectroscopic studies were performed (Figure 2). In the spectra for pristine material and intercalates, the peaks at 1380 and below $600 \mathrm{~cm}^{-1}$ are attributed to the $v\left(\mathrm{NO}_{3}{ }^{-}\right)$and $\nu(\mathrm{M}-\mathrm{O})$ modes. Symmetric and asymmetric stretching modes of sulfonates could be observed at around 1054 and $1200 \mathrm{~cm}^{-1}$, respectively, in the spectra of both $\mathrm{Na}^{+}$-taurine salt and CaM-Tau$\mathrm{LDH}$ intercalates. It is worthy to note that $v_{\text {asym }}\left(\mathrm{SO}_{3}{ }^{-}\right)$splits into two peaks at 1185 and $1223 \mathrm{~cm}^{-1}$ after intercalation (Figure 2(b)). Similar splitting of $\nu_{\text {asym }}\left(\mathrm{SO}_{3}{ }^{-}\right)$was reported in the infrared spectra of sulfonates coordinated to metal cations [27]. It is therefore concluded that the taurine molecules were stabilized in the interlayer space of CaMLDHs via coordination bonds between sulfonate and calcium cation.

From the X-ray diffraction patterns, FT-IR spectra and chemical formulae, we could suggest the possibility of an interlayer structure of taurine in an ideal case (Figure 3). According to the ICP-AES and CHNS elemental analysis, the chemical formulae of both CaM-Tau-LDHs were determined to be $\left[\mathrm{Ca}_{1.99} \mathrm{Al}(\mathrm{OH})_{6}(\mathrm{Tau})_{0.97} \cdot 5 \mathrm{H}_{2} \mathrm{O}\right]$ and $\left[\mathrm{Ca}_{1.91} \mathrm{Fe}(\mathrm{OH})_{6}(\mathrm{Tau})_{1.19} \cdot 5 \mathrm{H}_{2} \mathrm{O}\right]$, respectively. Since the amount of taurine molecules existing in the hybrids is almost the same compared to the ideal composition, $\mathrm{Ca}_{1.99} \mathrm{Al}$ $(\mathrm{OH})_{5.98}(\mathrm{Tau}) \cdot 5 \mathrm{H}_{2} \mathrm{O}$ and $\mathrm{Ca}_{1.91} \mathrm{Fe}(\mathrm{OH})_{5.82}(\mathrm{Tau}) \cdot 5 \mathrm{H}_{2} \mathrm{O}$, we could propose a schematic diagram of interlayer taurine orientation as shown in Figure 3.

In a previous study [26], we reported that the bond distance between calcium and the seventh coordinated nitrate is approximately $2.3 \AA$. The molecular dimensions along the $z$-axis for taurine (distance from outermost sulfate oxygen to amine hydrogen) and water are approximately 4.6 and $0.6 \AA$, respectively. Considering the van der Waals radii of hydrogen, the distance between the facing hydrogens is about $2 \AA$. For the summation of bond length $\mathrm{Ca}-\mathrm{O}$, the molecular dimensions along the $z$-axis and van der Waals interactions distance give $\sim 11.8 \AA$, which corresponds well to the basal spacing of CaM-Tau-LDHs determined by X-ray diffraction patterns. As suggested in Figure 3, the taurine and water molecules are arranged in a zig-zag pattern to be effectively stabilized in the interlayer space.

The particle size and morphology of pristine LDHs, intercalates, and $\mathrm{Na}^{+}$-taurine salts were investigated with scanning electron microscopy (Figure 4). Both CaM-LDHs before and after intercalation showed plate-like morphology with particle size distributed from 200 to $600 \mathrm{~nm}$, while $\mathrm{Na}^{+}$taurine salt morphology was undetermined, with particle sizes larger than $2 \mu \mathrm{m}$. In Figures $4(\mathrm{c})$ and $4(\mathrm{~d})$, the surface of CaM-Tau-LDHs seems to be covered with organic moieties which may have resulted from the surface-attached taurine molecules. It is worthy to note that the average particle size and morphology of the LDHs were not significantly altered 
after intercalation, which was also confirmed by the $\mathrm{X}$ ray diffraction result showing similar crystallinity for CaMLDHs and their intercalates (Figure 1).

It was also verified by the ninhydrin test that most of the taurine molecules were stabilized in the interlayer space of the CaM-LDHs. Ninhydrin molecules form deeppurple chromophores when they encounter primary amines. Figure 5 shows the UV-vis spectra and photographs of ninhydrin-treated $\mathrm{Na}^{+}$-taurine salt and CaM-Tau-LDHs. It should be noted that the amount of taurine in salt and intercalate was set at the same amount for quantitative analysis. As shown in the UV-vis spectra, the absorbance at $\lambda_{\max }=575 \mathrm{~nm}$ in intercalates was much lower than that of taurine salt, indicating that most of the taurine molecules did not react with the ninhydrin due to the stabilization of the LDH layers. The slightly brown color in the CaFe-Tau-LDH (Figure $5\left(\mathrm{e}^{\prime}\right)$ ) can be explained by the partial dissolution of $\mathrm{Fe}^{3+}$, which was also observed in the pristine $\mathrm{CaFe}-\mathrm{LDH}$ treated with ninhydrin (Figure 5( $\left.\mathrm{b}^{\prime}\right)$ ).

\section{Conclusion}

We have successfully intercalated the biologically active molecule taurine into two different kinds of calciumcontaining LDHs, CaAl- and CaFe-LDHs. Since coprecipitation and anionic exchange were determined to be not effective for the intercalation, we optimized the reaction conditions of a solid phase intercalation method. Structural analyses including X-ray diffraction patterns, infrared spectroscopy, microscopic study, ICP-AES, and ninhydrin tests showed that the taurine molecules were well stabilized between the LDH layers by forming direct coordination bonds with calcium cations.

\section{Acknowledgments}

This paper was financially supported by a National Research Foundation of Korea grant funded by the Korean Government (2010-0001488) and partly the Herbal Crop Research Project (PJ006921) of RDA, Republic of Korea.

\section{References}

[1] S. J. Choi, G. E. Choi, J. M. Oh, Y. J. Oh, M. C. Park, and J. H. Choy, "Anticancer drug encapsulated in inorganic lattice can overcome drug resistance," Journal of Materials Chemistry, vol. 20, no. 42, pp. 9463-9469, 2010.

[2] X. M. Jiang, Y. S. Cheng, and H. D. C. Smyth, "Nanostructured aerosol particles: fabrication, pulmonary drug delivery, and controlled release," Journal of Nanomaterials, vol. 2011, Article ID 198792, 2 pages, 2011.

[3] J. M. Oh, T. T. Biswick, and J. H. Choy, "Layered nanomaterials for green materials," Journal of Materials Chemistry, vol. 19, no. 17, pp. 2553-2563, 2009.

[4] E. Ruiz-Hernández, A. Baeza, and M. Vallet-Regí, "Smart drug delivery through DNA/magnetic nanoparticle gates," ACS Nano, vol. 5, no. 2, pp. 1259-1266, 2011.

[5] L. X. Zhao, A. C. Liu, M. Sun et al., "Enhancement of oral bioavailability of puerarin by polybutylcyanoacrylate nanoparticles," Journal of Nanomaterials, vol. 2011, Article ID 126562, 8 pages, 2011.

[6] A. Vaccari, "Preparation and catalytic properties of cationic and anionic clays," Catalysis Today, vol. 41, no. 1-3, pp. 53-71, 1998.

[7] J. H. Choy, J. S. Jung, J. M. Oh et al., "Layered double hydroxide as an efficient drug reservoir for folate derivatives," Biomaterials, vol. 25, no. 15, pp. 3059-3064, 2004.

[8] J. H. Choy, S. Y. Kwak, J. S. Park, Y. J. Jeong, and J. Portier, "Intercalative nanohybrids of nucleoside monophosphates and DNA in layered metal hydroxide," Journal of the American Chemical Society, vol. 121, no. 6, pp. 1399-1400, 1999.

[9] J. M. Oh, M. Park, S. T. Kim, J. Y. Jung, Y. G. Kang, and J. H. Choy, "Efficient delivery of anticancer drug MTX through MTX-LDH nanohybrid system," Journal of Physics and Chemistry of Solids, vol. 67, no. 5-6, pp. 1024-1027, 2006.

[10] L. van der Ven, M. L. M. van Gemert, L. F. Batenburg et al., "On the action of hydrotalcite-like clay materials as stabilizers in polyvinylchloride," Applied Clay Science, vol. 17, no. 1-2, pp. 25-34, 2000.

[11] K. M. Kim, C. B. Park, A. J. Choi, J. H. Choy, and J. M. Oh, "Selective DNA adsorption on layered double hydroxide nanoparticles," Bulletin of the Korean Chemical Society, vol. 32, no. 7, pp. 2217-2221, 2011.

[12] W. Kagunya, Z. Hassan, and W. Jones, "Catalytic properties of layered double hydroxides and their calcined derivatives," Inorganic Chemistry, vol. 35, no. 21, pp. 5970-5974, 1996.

[13] J. M. Oh, S. Y. Kwak, and J. H. Choy, "Intracrystalline structure of DNA molecules stabilized in the layered double hydroxide," Journal of Physics and Chemistry of Solids, vol. 67, no. 5-6, pp. 1028-1031, 2006.

[14] J. M. Oh, S. J. Choi, G. E. Lee, J. E. Kim, and J. H. Choy, "Inorganic metal hydroxide nanoparticles for targeted cellular uptake through clathrin-mediated endocytosis," Chemistry, vol. 4, no. 1, pp. 67-73, 2009.

[15] J. M. Oh, S. J. Choi, S. T. Kim, and J. H. Choy, "Cellular uptake mechanism of an inorganic nanovehicle and its drug conjugates: enhanced efficacy due to clathrin-mediated endocytosis," Bioconjugate Chemistry, vol. 17, no. 6, pp. 14111417, 2006.

[16] S. J. Choi, J. M. Oh, and J. H. Choy, "Toxicological effects of inorganic nanoparticles on human lung cancer A549 cells," Journal of Inorganic Biochemistry, vol. 103, no. 3, pp. 463-471, 2009.

[17] S. J. Choi, J. M. Oh, and J. H. Choy, "Biocompatible ceramic nanocarrier for drug delivery with high efficiency," Journal of the Ceramic Society of Japan, vol. 117, no. 1365, pp. 543-549, 2009.

[18] J. M. Oh, S. J. Choi, G. E. Lee, S. H. Han, and J. H. Choy, "Inorganic drug-delivery nanovehicle conjugated with cancercell-specific ligand," Advanced Functional Materials, vol. 19, no. 10, pp. 1617-1624, 2009.

[19] V. Ambrogi, G. Fardella, G. Grandolini, and L. Perioli, "Intercalation compounds of hydrotalcite-like anionic clays with antiinflammatory agents-I. Intercalation and in vitro release of ibuprofen," International Journal of Pharmaceutics, vol. 220, no. 1-2, pp. 23-32, 2001.

[20] S. J. Lippard and J. M. Berg, Princlples of Bioinorganic Chemistry, University Science Book, Mill Valley, Calif, USA, 1994.

[21] G. Renaudin and M. François, "The lamellar doublehydroxide $(\mathrm{LDH})$ compound with composition $3 \mathrm{CaO} \cdot \mathrm{Al} 2 \mathrm{O} 3$. $\mathrm{Ca}(\mathrm{NO} 3) 2 \cdot 10 \mathrm{H} 2 \mathrm{O}$," Acta Crystallographica Section C, vol. 55, no. 6 , pp. $835-838,1999$. 
[22] T. R. Green, J. H. Fellman, A. L. Eicher, and K. L. Pratt, "Antioxidant role and subcellular location of hypotaurine and taurine in human neutrophils," Biochimica et Biophysica Acta, vol. 1073, no. 1, pp. 91-97, 1991.

[23] N. Ozmeric, G. Ozcan, C. M. Haytac, E. E. Alaaddinoglu, M. F. Sargon, and S. Senel, "Chitosan film enriched with an antioxidant agent, taurine, in fenestration defects," Journal of Biomedical Materials Research, vol. 51, pp. 500-503, 2000.

[24] J. Plank and F. von Hoessle, "Formation of an inorganicorganic host-guest material by intercalation of acetone formaldehyde sulfite polycondensate into a hydrocalumite structure," Zeitschrift fur Anorganische und Allgemeine Chemie, vol. 636, no. 8, pp. 1533-1537, 2010.

[25] A. Hayashi and H. Nakayama, "Simple intercalation reaction of layered double hydroxide with sodium valproate under solid conditions," Chemistry Letters, vol. 39, no. 10, pp. 10601062, 2010.

[26] T. H. Kim, I. Heo, C. B. Park et al., "Layered metal hydroxides containing calcium and their structural analysis," Bulletin of the Korean Chemical Society, vol. 33, no. 6, pp. 1845-1850, 2012.

[27] B. Sun, Y. Zhao, J. G. Wu, Q. C. Yang, and G. X. Xu, "Crystal structure and FT-IR study of cesium 4-methylbenzenesulfonate," Journal of Molecular Structure, vol. 471, no. 1-3, pp. 63-66, 1998. 

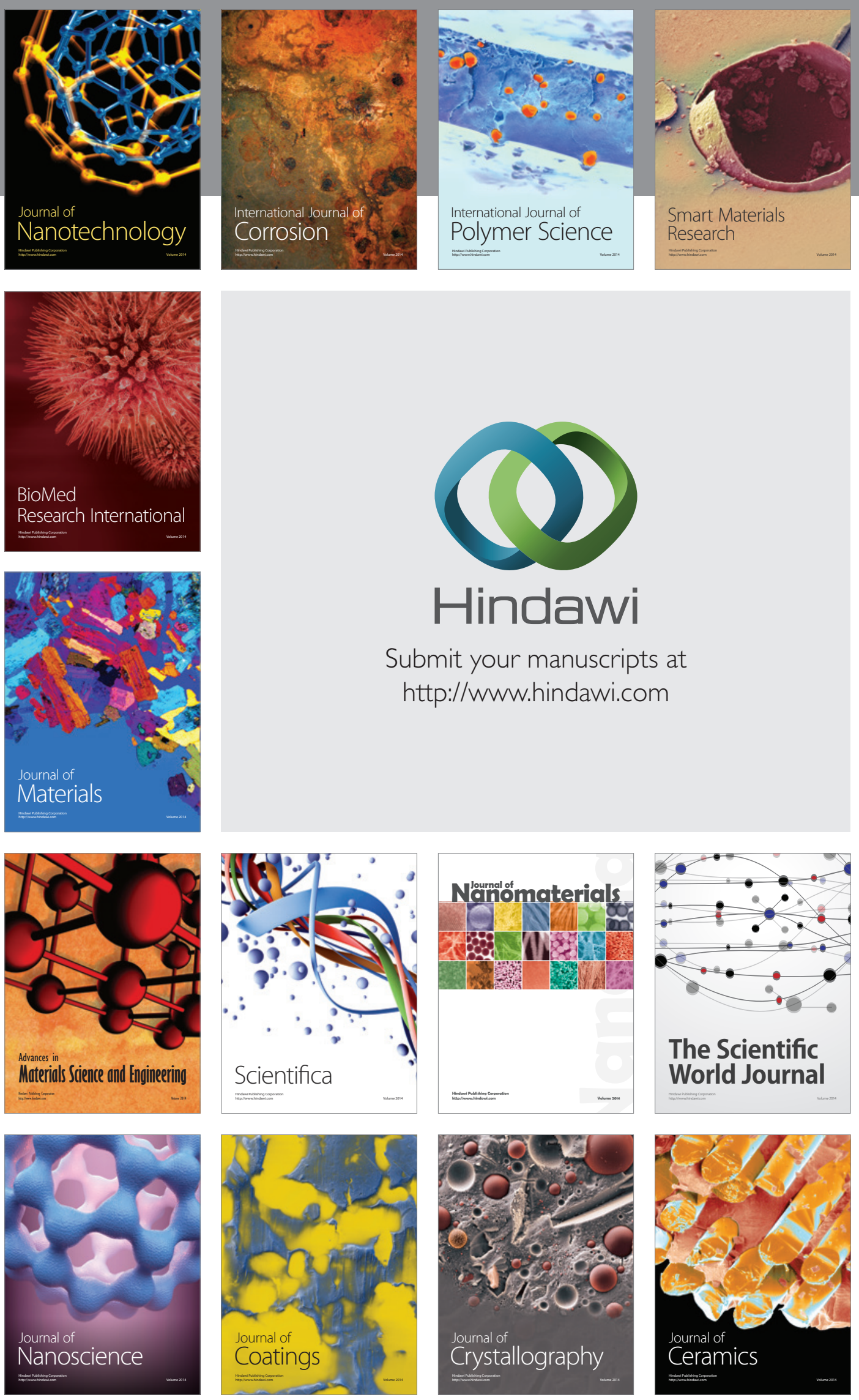

The Scientific World Journal

Submit your manuscripts at

http://www.hindawi.com

\section{World Journal}

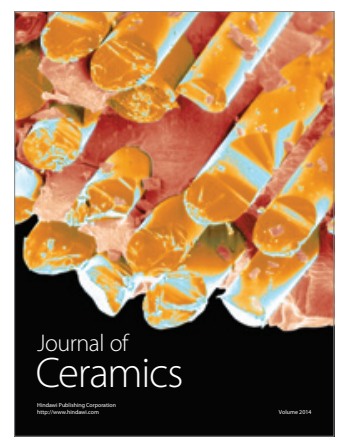

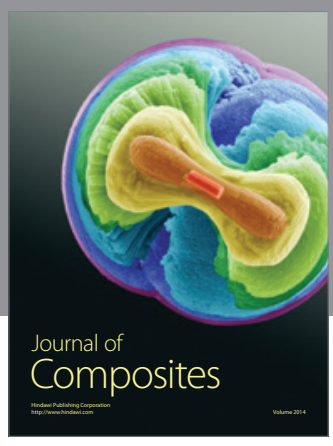
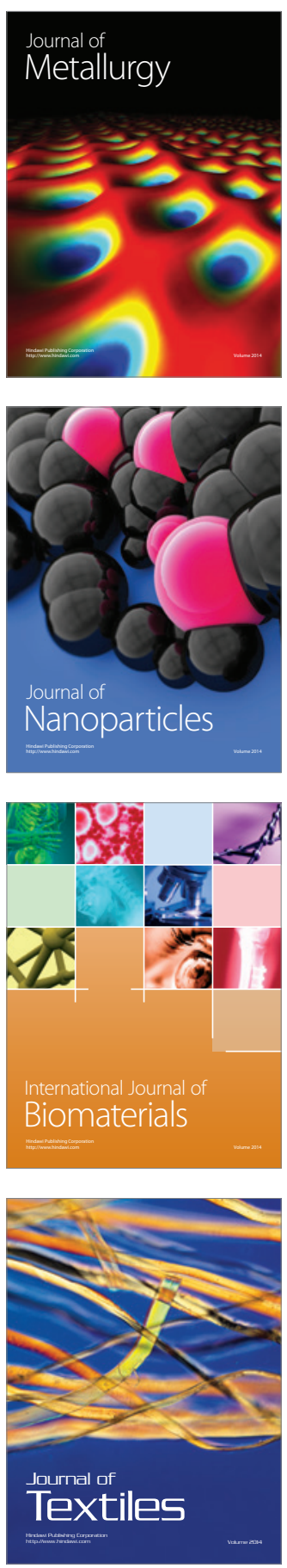